\title{
Time-Independent Density-Functional Theory for Excited States of Coulomb Systems
}

\author{
Paul W. Ayers, ${ }^{1}$ Mel Levy ${ }^{2-4}$, and Agnes Nagy ${ }^{5}$ \\ 1. Department of Chemistry and Chemical Biology, McMaster University; Hamilton, Ontario, Canada L8S 4M1 \\ 2. Department of Physics, North Carolina A\&T State University; Greensboro NC 27411 \\ 3. Department of Chemistry, Duke University, Durham NC 27708 \\ 4. Department of Chemistry, Tulane University, New Orleans LA 70118 \\ 5. Department of Theoretical Physics, University of Debrecen, H-4010 Debrecen, Hungary
}

\begin{abstract}
A Coulomb density is special because it determines not only its Hamiltonian but the degree of excitation as well. We derive Euler equations for excited state energies and densities that depend only on the electron density. Unlike existing formulations, additional functions and indices are not required; with these functionals, the equations of excited-state density functional theory strongly resemble those of ground-state theory. A critical analysis of the new functionals is included.
\end{abstract}

\section{Introduction}

The thermodynamic, electronic, and spectroscopic properties of atoms, molecules, and materials are determined by the relative energies and electronic properties of the ground and excited states. Consequently, computational methods for characterizing the spectrum of energy levels play a pivotal role in the description of experimental observations and the prediction of new experimental phenomena. Unfortunately, the most straightforward approach to characterizing electronic systems-i.e., solving the many electron Schrödinger equation-is 
impractical except for very small systems, because the wavefunction's complexity grows rapidly with increasing size. More practical approaches to the electronic structure problem can be formulated using the electron density which, unlike the wavefunction, is always threedimensional. With this in mind, Hohenberg, Kohn, and Sham ${ }^{1,2}$ ushered in modern densityfunctional theory (DFT) for nondegenerate ground states. (See ref. 3 for a history of the extension of ground-state DFT to degeneracies through the constrained search.) It soon became apparent that it would be important to extend DFT to describe excited states, which is an area of active research. The overwhelming majority of excited-state calculations, thus far, have utilized the time-dependent approach, where information about excited states is extricated from the response of the system to time-dependent perturbations. (See, for instance, references ${ }^{4-7}$.) In conventional wavefunction-based quantum mechanics, however, individual excited states arise as stationary states of the energy, and no reference to time-dependence is necessary. To many, then, a most natural approach to excited-state DFT is one that uses time-independent density functionals ${ }^{8,9}$ where the individual excited-state energies and electron densities arise as stationary states of an energy expression of the form

$$
E[\rho]=\int \rho(\mathbf{r}) v(\mathbf{r}) d \mathbf{r}+F[\rho]
$$

where $v(\mathbf{r})$ is the external potential (the attractive one-body potential of interest), $\rho(\mathbf{r})$ is the electron density, and $F[\rho]$ is sum of the kinetic and electron-electron repulsion energies, expressed as a functional of $\rho(\mathbf{r})$.

Equation (1) is precisely the form of the energy expression for the ground state. But does an analogous equation exist for excited states? A main purpose of this article is to give an affirmative answer for molecular systems when $F[\rho]$ explicitly incorporates the fact that $v(\mathbf{r})$ is a 
Coulomb potential. Specifically, we show that there exists, with certain caveats, a universal functional $F^{C o u l}[\rho]$ such that

$$
\frac{\delta}{\delta \rho(\mathbf{r})}\left\{\int v^{\text {Coul }}(\mathbf{r}) \rho(\mathbf{r}) d \mathbf{r}+F^{\text {Coul }}[\rho]\right\}_{\rho=\rho_{k}}=\text { constant }
$$

with

$$
E_{k}=\int v^{\text {Coul }}(\mathbf{r}) \rho_{k}(\mathbf{r}) d \mathbf{r}+F^{\text {Coul }}\left[\rho_{k}\right]
$$

where $E_{k}$ is the energy of the $k^{\text {th }}$ stationary state of Coulomb potential $v^{\operatorname{Coul}}(\mathbf{r})$, with corresponding density $\rho_{k}(\mathbf{r})$. Note that $k=1$ denotes the ground state. So, with a single $F$, Eq. (2) is appropriate for the ground state as well as for all bound excited states. One functional does it all. (We note in passing that there are special cases where the ground-state constrained-search $F$ yields exact excited-state energies. ${ }^{10}$ )

We shall also introduce and analyze the functional $F_{k}^{\text {Coul }}[\rho]$. The corresponding Euler equation with $F_{k}^{\text {Coul }}[\rho]$ is Eq. (2), but with $F^{\text {Coul }}$ simply replaced by $F_{k}^{\text {Coul }}$. That is,

$$
\frac{\delta}{\delta \rho(\mathbf{r})}\left\{\int v^{\text {Coul }}(\mathbf{r}) \rho(\mathbf{r}) d \mathbf{r}+F_{k}^{\text {Coul }}[\rho]\right\}_{\rho=\rho_{k}}=\text { constant }
$$

with

$$
E_{k}=\int v^{\text {Coul }}(\mathbf{r}) \rho_{k}(\mathbf{r}) d \mathbf{r}+F_{k}^{\text {Coul }}\left[\rho_{k}\right]
$$

Unlike $F^{\text {Coul }}$, the functional $F_{k}^{\text {Coul }}$ explicitly incorporates $k$, the excitation label of the state of interest. Both $F^{\mathrm{Coul}}[\rho]$ and $F_{k}^{\mathrm{Coul}}[\rho]$ will be defined for any well-behaved density, whether that density is Coulombic or not. However, when the attractive potential $v(\mathbf{r})$ is not Coulombic, the solutions to the Euler equations will not necessarily correspond to stationary states. 
In contrast to $F^{C o u l}[\rho]$ and $F_{k}^{\text {Coul }}[\rho]$, previous $F$ 's for excited states, though certainly encouraging, have had to utilize an ensemble of states ${ }^{11-18}$ or have had to depend on additional information besides $\rho(\mathbf{r})$, such as the incorporation of the external potential or its ground-state density (to form a bifunctional), ${ }^{19-24}$ in order to take into consideration orthogonality requirements to lower states during energy minimization. The formulation of Ziegler et al., optimizes both the density and a unitary matrix, which imposes orthogonality at the Kohn-Sham level. ${ }^{25}$

It has been shown that there exists no $F[\rho]$ for excited states such that the minimization ${ }^{26}$ of $E[\rho]$ in Eq. (1) would guarantee exact excited-state energies, even for Coulomb systems. With this in mind, Görling ${ }^{27}$ derived a DFT stationary principle for excited states that bypasses an energy minimization. His formulation has strongly influenced our development of equations (2) and (3). Specifically, he put forth an equation analogous to Eq. (3), but not with $F_{k}^{\text {Coul }}[\rho]$. Instead, although our $F_{k}^{\text {Coul }}[\rho]$ is in the spirit of Görling's functional in that his also depends on an index in addition to $\rho(\mathbf{r})$, the important difference is that his index $v$, unlike the $k$ in $F_{k}^{\text {Coul }}[\rho]$, does not necessarily correspond to the level of excitation under consideration. In other words, his $v=3$ might correspond to the first excited state, etc.. In contrast, if the first excited-state energy is desired, one simply inserts $k=g+1$ into $F_{k}^{\text {Coul }}[\rho]$, where $g$ is order of the degeneracy of the ground state. As will be seen, this follows from the fact that our formulation specifically utilizes the fact that a Coulomb system is under consideration-that is, $v(\mathbf{r})=v^{\operatorname{Coul}}(\mathbf{r})$.

That Eqs. (2) and (3) exist might be surprising to most readers in view of the fact that it is well known that an excited-state density of one Hamiltonian is commonly a ground-state density of another Hamiltonian. Even more relevant, several authors have provided theoretical and 
numerical evidence that a given density might be an eigendensity associated with the $k^{\text {th }}$ state of several different external potentials. ${ }^{28-31}$ Consequently, it would appear that Eqs. (2) and (3) are not valid, since they require that each solution, $\rho_{k}(\mathbf{r})$, be associated with only one external potential. This indicates the path to a possible solution: find a set of external potentials, encompassing as many as possible of the external potentials of "practical" interest, and construct a density-functional theory on this limited set of potentials. With this in mind, we shall now show that Eqs. (2) and (3) are indeed valid for excited states because $v(\mathbf{r})=v^{\operatorname{Coul}}(\mathbf{r})$ in these equations, and there is something special about Coulomb external potentials.

\section{Theory}

For our development, assume that the Coulomb Hamiltonian of interest is

$$
\hat{H}^{\text {Coul }}=\sum_{i=1}^{N} v^{\text {Coul }}\left(\mathbf{r}_{i}\right)+\hat{T}+\hat{V}_{e e},
$$

where $\hat{T}$ is the kinetic energy operator, $\hat{V}_{e e}$ is the electron-electron repulsion operator, and

$$
v^{\text {Coul }}(\mathbf{r})=\sum_{\alpha=1}^{M} \frac{-Z_{\alpha}}{\left|\mathbf{r}-\mathbf{R}_{\alpha}\right|}
$$

where $Z_{\alpha}$ is the charge on nucleus $\alpha, \mathbf{r}$ is the position of the electron, $\mathbf{R}_{\alpha}$ is the position of the nucleus (as represented by a discrete point charge), and $M<\infty$ is the number of nuclei. Our results will follow from the fact that a $\rho(\mathbf{r})$ is allowed to be an eigendensity for at most one $v^{\text {Coul }}(\mathbf{r})$ and for only one of that system's states. In other words, we shall utilize our realization that Coulomb densities determine not only the external potential, but also the degree of excitation of the system. 
Building on the arguments of E. B. Wilson for Coulomb ground states, Nagy formulated an excited-state $\mathrm{DFT}^{32}$ for Coulomb systems using the realization that the density of a Coulomb stationary state (whether excited or ground) determines the positions and charges of the atomic nuclei (therefore $v^{\text {Coul }}(\mathbf{r})$ ) through conventional cusp conditions ${ }^{33-35}$ or, in cases where there is no electron density at the nucleus, generalizations thereof. ${ }^{36-39}$ Theorem I summarizes these results:

\section{Theorem I:}

Let $\rho(\mathbf{r})$ be the electron density of a Coulomb system, that is, a system whose external potential can be written in the form of Eq. (5). Then $\rho(\mathbf{r})$ is not a stationary state wave function for any other Coulomb external potential.

Theorem I is, by itself, not enough to construct an excited-state density-functional theory for Coulomb systems, because it does not exclude the possibility that two different excited states of the same system might have the same electron density. However, observe that this possibility is indeed excluded because the asymptotic decay of the electron density in a Coulomb system is given by ${ }^{40-45}$

$$
\lim _{r \rightarrow \infty} \frac{\partial \ln \bar{\rho}_{k}(\mathbf{r})}{\partial r}=-\sqrt{8\left(E_{1}^{(N-1)}-E_{k}^{(N)}\right)},
$$

unless forbidden by symmetry, where $\bar{\rho}_{k}(\mathbf{r})$ is the spherically averaged density for the $k^{\text {th }}$ state of the $N$-electron system, $E_{1}^{(N-1)}$ is the ground state energy of the $N-1$ electron system, and $E_{k}^{(N)}$ is the energy of the $k^{\text {th }}$ state of the $N$-electron system. Because $E_{1}^{(N-1)}$ is independent of $k$, it follows from Eq. (6) that $\rho_{k}(\mathbf{r})$ has a unique $E_{k}^{(N)}$. Moreover, because the external potential is known from Theorem I, and because the number of electrons, $N$, is known from direct 
integration of $\rho_{k}(\mathbf{r})$, we can determine the energy associated with any eigendensity of any Coulomb system directly from Eq. (6). ${ }^{36}$ With this information, we can then compute $F^{\text {Coul }}[\rho]$ for this system directly from the definition

$$
F^{\text {Coul }}[\rho] \equiv E_{k}[\rho]-\int \rho(\mathbf{r}) v^{\text {Coul }}[\rho ; \mathbf{r}] d \mathbf{r}
$$

Equation (7) is valid for any bound stationary state of any system with a Coulomb external potential, subject only to the caveat that Eq. (6) holds. Equation (6) holds when the Dyson amplitude, $\quad g_{k}=\left\langle\Psi_{1}^{(N-1)} \mid \Psi_{k}^{(N)}\right\rangle$, is not zero. This happens, for example, when the spinmultiplicity of the excited state and the ground-state ion differ by more than one..$^{41-43,46}$ In all cases, a large manifold of bound excited states can be obtained from this functional; in the absence of spatial symmetry it seems likely that the only missing excited states are those associated with an extra spin-flip, relative to the ground-state ion. The key relation, Eq. (6), also holds for unbound resonances. However, we will not address unbound excited states except to note that recent results from the literature suggest that our results could be extended to resonances using complex scaling. ${ }^{47,48}$

It is important to emphasize that this entire analysis is predicated on the fact that the given density, $\rho(\mathbf{r})$, is known to be Coulombic. Given an arbitrary density, this method will fail, since there is no known way to determine whether the given density is Coulombic without constructing the predicted external potential from step 1 of the above algorithm, solving the Schrödinger equation for this system, and then explicitly testing whether or not the given density is associated with one of the eigenstates. In particular, there are many densities possessing the sorts of cusps and asymptotic decay that typify a Coulomb density that are not themselves Coulombic. For example, given an atomic density, $\rho_{\text {atom }}(\mathbf{r})$, the electron density 


$$
\tilde{\rho}(\mathbf{r})=\rho_{\text {atom }}(\mathbf{r})+\varepsilon r^{2}\left(r^{2}-1\right) e^{-\frac{3}{2} r^{2}},
$$

( $\varepsilon$ is any number for which $\tilde{\rho}(\mathbf{r})$ remains positive) has the same number of electrons, the same cusp, and the same asymptotic decay as $\rho_{\text {atom }}(\mathbf{r})$. Yet the revised density, $\tilde{\rho}(\mathbf{r})$, is not Coulombic.

Since electron densities that appear Coulombic might not be, the approach proposed in the previous section possesses what may be termed the Coulombic $v$-representable problem; namely, there is no good way to discern whether a given density is an eigendensity for a Coulomb system. Fortunately, we can avoid this problem by defining a functional that exists for all electron densities, even if they are not Coulombic.

In conventional ground-state DFT, this objective is usually performed using the constrained search. ${ }^{3}$ Accordingly, consider the following definition:

Definition: Let $\rho(\mathbf{r})$ be any trial $N$-representable electron density and let $\rho^{\text {Coul }}(\mathbf{r})$ be a Coulomb density corresponding to the $k^{\text {th }}$ eigenstate of some Coulomb Hamiltonian, with external potential $v^{\text {Coul }}\left[\rho^{\text {Coul }} ; \mathbf{r}\right]$. The first $k-1$ eigenstates of this Hamiltonian are denoted $\left\{\Psi_{n}^{\text {Coul }}\left[\rho^{\text {Coul }} ; \mathbf{r}\right]\right\}_{n=1}^{k-1}$. We then define

$$
\begin{aligned}
& F\left[\rho, \rho^{\text {Coul }}\right]=\min \left\langle\Psi\left|\hat{T}+\hat{V}_{e e}\right| \Psi\right\rangle \\
& \text { subject to: } \Psi \rightarrow \rho(\mathbf{r}),\left\{\left\langle\Psi \mid \Psi_{n}^{\text {Coul }}\left[\rho^{\text {Coul }}\right]\right\rangle=0\right\}_{n=1}^{k-1}
\end{aligned}
$$

That is, we minimize the sum of the kinetic and electron-electron energies, subject to the constraint that each $\Psi$ yields $\rho(\mathbf{r})$ and is simultaneously 
orthogonal to the first $k-1$ states of the Coulomb system specified by

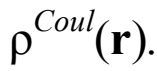

As stated, $F\left[\rho, \rho^{\mathrm{Coul}}\right]$ is a bifunctional, with a form similar to the Levy-Nagy bifunctional. ${ }^{19}$ In order to obtain a universal functional that depends only on the electron density, we need to write $\rho^{\text {Coul }}(\mathbf{r})$ as a functional of the electron density, $\rho(\mathbf{r})$. Intuitively, it seems reasonable to establish this linkage by choosing $\rho^{\text {Coul }}(\mathbf{r})$ to be the Coulomb density that is closest to $\rho(\mathbf{r})$. (The distance can be measured using the $\mathbb{L}^{1}$ norm or, better still, as

$$
\begin{aligned}
d_{\mathbb{H I}^{1}}\left(\rho^{\text {Coul }}, \rho\right) \equiv & \equiv \int\left(\sqrt{\rho^{\text {Coul }}(\mathbf{r})-\rho(\mathbf{r})}\right)^{2} d \mathbf{r} \\
& +\int \nabla \sqrt{\rho^{\text {Coul }}(\mathbf{r})-\rho(\mathbf{r})} \cdot \nabla \sqrt{\rho^{\text {Coul }}(\mathbf{r})-\rho(\mathbf{r})} d \mathbf{r}
\end{aligned}
$$

In this work, we will not dwell on the nuances of choosing the best measure for the distance.)

This approach assumes that there is a unique Coulomb density, $\rho^{C o u l}[\rho ; \mathbf{r}]$ that is closest to the non-Coulomb $\rho(\mathbf{r})$ in question. If there are several Coulomb densities that are the same minimum distance from $\rho(\mathbf{r})$, then we can choose Eq. (9) to be the smallest attainable value of $F\left[\rho, \rho^{C o u l}\right]$. Mathematically, we construct this functional in the following way. First, we define the functional,

$$
F_{\varepsilon}^{\text {Coul }}[\rho]=\underbrace{\min }_{\rho(\mathbf{r})} F\left[\rho, \rho^{\text {Coul }}\right] \text { where }\left\|\rho^{\text {Coul }}-\rho(\mathbf{r})\right\| \leq \varepsilon
$$

where $\varepsilon$ is large enough to ensure there exists at least one stationary state Coulomb density, $\rho^{\text {Coul }}(\mathbf{r})$, within $\varepsilon$ units of $\rho^{\text {Coul }}(\mathbf{r})$. Let $\varepsilon_{\min }$ denote the smallest attainable value of $\varepsilon$ in Eq. (11). $F^{C o u l}[\rho]$ is simply

$$
F^{\text {Coul }}[\rho]=F_{\varepsilon_{\min }}^{\text {Coul }}[\rho]
$$


Let's now generate $F_{k}^{\text {Coul }}[\rho]$. For this purpose, first consider $F_{\varepsilon, k}^{\text {Coul }}[\rho]$, which we define as

$$
F_{\varepsilon, k}^{\text {Coul }}[\rho]=\underbrace{\min }_{\rho_{k}^{\text {Coul }}} F\left[\rho, \rho_{k}^{\text {Coul }}\right] \text { where }\left\|\rho_{k}^{\text {Coul }}-\rho(\mathbf{r})\right\| \leq \varepsilon
$$

where $\varepsilon$ is large enough to ensure there exists at least one $k^{\text {th }}$ stationary state Coulomb density, $\rho_{k}^{\text {Coul }}(\mathbf{r})$ within $\varepsilon$ units of $\rho(\mathbf{r}) . F_{k}^{\text {Coul }}[\rho]$ is then given by

$$
F_{k}^{\text {Coul }}[\rho]=F_{\varepsilon_{\min }, k}^{\text {Coul }}[\rho]
$$

In other words, we find the closest density to $\rho(\mathbf{r})$ that is the $k^{\text {th }}$ stationary state density for a Coulomb system, and then construct $F_{k}^{\text {Coul }}[\rho]$ using wavefunctions that are orthogonal to the states of that Coulomb system. (If several $k^{\text {th }}$-state Coulomb densities are the same minimal distance from the density of interest, we select the Coulomb system that yields the smallest value for $F_{k}^{\text {Coul }}[\rho]$.) Incidentally, ensembles could be used instead of pure states in the constrainedsearch definitions of both $F^{\text {Coul }}[\rho]$ and $F_{k}^{\text {Coul }}[\rho]$.

Finally, following the reasoning of Görling, ${ }^{27}$ we observe that Eqs. (2) and (3) arise from the analogous stationary principle involving the Schrödinger equation. Namely,

$$
\frac{\delta}{\delta \Psi}\left\{\int v(\mathbf{r}) \rho[\psi, \mathbf{r}] d \mathbf{r}+\left\langle\Psi\left|\hat{T}+\hat{V}_{e e}\right| \Psi\right\rangle\right\}_{\Psi=\Psi_{k}}=\text { constant }
$$

where, in accordance with their definitions, the $\Psi$ 's in Eq. (15) are the wavefunctions associated with $\varepsilon_{\min }$ in either Eq. (12) or (14).

The constrained-search formulation formally addresses the problem of Coulombic- $v$ representability, but it does not guarantee that the functionals are well-behaved. In particular, we do not know if $F^{\operatorname{Coul}}[\rho]$ and $F_{k}^{\text {Coul }}[\rho]$ are functionally differentiable or, for that matter, even if 
they are continuous. It is important, then, to attempt to characterize these functionals. This requires a mathematical analysis of the structure of the set of Coulomb external potentials and the associated electron densities, which is provided in the appendix.

We conclude with a summary of the preceding arguments. Because no two stationary states of Coulomb systems can have the same electron density, it follows that a Coulomb density determines not only the external potential, but also the excitation level of the Coulomb external potential to which it corresponds. This lays the foundation for the universally defined excitedstate functionals, $F^{C o u l}[\rho]$ and $F_{k}^{C o u l}[\rho]$, which give exact results for Coulomb systems like molecules. Although there is no practical general method for determining whether a given electron density is an eigendensity for a Coulomb system (this may be termed the Coulombic- $v$ representability problem), it is fortunate that, just as in conventional DFT, the Coulombic- $v$ representable problem can be circumvented via constrained search. We show two ways of doing this, obtaining the functionals $F^{\text {Coul }}[\rho]$ (cf. Eq. (12)) and $F_{k}^{\text {Coul }}[\rho]$ (cf. Eq. (14)). The former functional is particularly enticing because it is stationary for every eigendensity of every Coulomb system; that is, this functional is the most direct analogue to the stationarity principle for excited-state wave functions in wavefunction-based quantum mechanics. However, it is possible that $F^{C o u l}[\rho]$ is a jagged, discontinuous functional: this could occur if extremely similar Coulomb densities have very different values of $F^{C o u l}[\rho]$, so that $F^{C o u l}[\rho]$ would be discontinuous (or, less severely, barely continuous). The functional $F_{k}^{\text {Coul }}[\rho]$, on the other hand, is somewhat less ambitious, since it has an additional dependence on the level of excitation, $k$. However, because the level of excitation in $F_{k}^{\text {Coul }}[\rho]$ is explicitly accommodated, discontinuities are less 
likely and its properties for approximation purposes are more transparent (e.g., $\left.F_{k+1}^{\text {Coul }}[\rho] \geq F_{k}^{\text {Coul }}[\rho]\right)$

Computational work in time-independent excited-state DFT is based upon the KohnSham formulation, ${ }^{19-21,27,30,31,49-57}$ and practical applications of the present functionals also demand the formulation of a Kohn-Sham theory. We will describe a Kohn-Sham theory involving $F_{k}^{\text {Coul }}[\rho]$ in a future publication. The basic idea is that the KS kinetic energy may be defined by minimizing $\langle\Psi|\hat{T}| \Psi\rangle$ with respect to all wavefunctions that (a) yield $\rho(\mathbf{r})$ and (b) are orthogonal to the first $k-1$ states of the non-interacting Hamiltonian with the same ground-state density as the interacting Coulomb Hamiltonian whose $k^{\text {th }}$ state density is associated with $\varepsilon_{\min }$ in Eq. (14). The corresponding non-Coulombic Kohn-Sham effective potential is generated in a manner that is analogous to that of the ground state and the resulting orbitals form the $k^{\text {th }}$ state of the effective potential.

\section{Concluding Thoughts}

Universal functionals are the theoretical foundation for present-day approaches to DFT. However, among the entire universe of possible systems, only a tiny galaxy of them are relevant to molecular science. This motivates the search for subuniversal functionals. ${ }^{58-60}$ Based on the favorable formal characteristics presented here, subuniversal functionals focused on Coulomb systems are certainly worthy of further investigation. The functionals $F^{\text {Coul }}[\rho]$ and $F_{k}^{\text {Coul }}[\rho]$ are universal in the sense that they are defined for all well-behaved electron densities, independent of the external potential under consideration. However, we classify these functionals as subuniversal because they yield exact excited-state energies and densities only for Coulomb 
potentials. It will be particular important to rigorously characterize the continuity and differentiability of these functionals, to explore different methods for characterizing Coulomb densities and external potentials, and to formulate noninteracting reference systems that can be used in practical calculations. Most generally, however, this work serves to emphasize that subuniversal functionals could be useful tools in excited-state DFT.

\section{Acknowledgements:}

Partial support for this work was provided by NSF, NSERC, and the Canada Research Chairs. The work is also supported by Grant OTKA No. K 100590 and TAMOP 4.2.1/B09/1/KONV-2010-0007 and the TAMOP 4.2.2/B-10/1-2010-0024 projects. The project is cofinanced by the European Union and the European Social Fund. 


\section{Appendix.}

The following analysis seeks to analyze the properties of the functionals $F^{{ }^{C o u l}}[\rho]$ and $F_{k}^{\text {Coul }}[\rho]$. In particular, it is important that the functionals be continuous, since it probably would be very difficult to construct useful approximations to a discontinuous functional. This requires a mathematical analysis of the structure of the set of Coulomb external potentials and the associated electron densities, which is provided as supplementary material.

To begin, consider that many (if not all) external potentials of interest can be viewed as being generated by some distribution of electric charge, $\mathcal{P}(\mathbf{r}),{ }^{61}$

$$
v(\mathbf{r})=\int \frac{\mathcal{P}\left(\mathbf{r}^{\prime}\right)}{\left|\mathbf{r}-\mathbf{r}^{\prime}\right|} d \mathbf{r}^{\prime}
$$

We call these external potentials "generalized Coulomb external potentials" to distinguish them from the special case where $\mathcal{P}\left(\mathbf{r}^{\prime}\right)$ is an assemblage of point charges as in Eq. (5)

Evaluating Eq. (A1) using a $K$-point quadrature formula,

$$
v_{K}^{\text {Coul }}(\mathbf{r}) \equiv \sum_{i=1}^{K} w_{i} \frac{\mathcal{P}\left(\mathbf{r}_{i}^{\prime}\right)}{\left|\mathbf{r}-\mathbf{r}_{i}^{\prime}\right|}=\sum_{i=1}^{K} \frac{q_{i}}{\left|\mathbf{r}-\mathbf{r}_{i}^{\prime}\right|} \approx v(\mathbf{r})
$$

where the charge, $q_{i}$, is identified with $w_{i} \mathcal{P}\left(\mathbf{r}_{i}^{\prime}\right)$. It follows from Eq. (A2) that by choosing an appropriately large value of $K$, one may approximate — to arbitrary accuracy — any generalized Coulomb external potential with a system of point charges. ${ }^{62}$ The nature of this approximation is peculiar, and requires further study. Note that in Eq. (A2) there are a few places (specifically, the $K$ points $\left.\left\{\mathbf{r}_{i}^{\prime}\right\}_{i=1}^{K}\right)$ where the Coulomb potential diverges to infinity, but the target potential, $v(\mathbf{r})$, generally does not diverge. That is, while the approximation in Eq. (A2) may be very accurate almost everywhere, but where it fails it can be extraordinarily poor. This is most readily apparent when one takes the Laplacian of both sides of Eq. (A2), obtaining 


$$
\mathcal{P}\left(\mathbf{r}^{\prime}\right) \not \sum_{i=1}^{K} q_{i} \delta\left(\mathbf{r}^{\prime}-\mathbf{r}_{i}^{\prime}\right)
$$

On the other hand, for functions $f(\mathbf{r})$ that are sufficiently well behaved,

$$
\int f(\mathbf{r}) v_{K}^{\text {Coul }}(\mathbf{r}) d \mathbf{r}=\sum_{i=1}^{K} \int f(\mathbf{r})\left(\frac{q_{i}}{\left|\mathbf{r}-\mathbf{r}_{i}^{\prime}\right|}\right) d \mathbf{r} \approx \int f(\mathbf{r}) v(\mathbf{r}) d \mathbf{r}
$$

with the approximation converging to an equality as $K \rightarrow \infty$.

Suppose $\rho(\mathbf{r})$ is an ensemble- $\boldsymbol{v}$-representable density corresponding to a stationary state of the $N$-electron system with generalized Coulomb external potential $v(\mathbf{r})$, as per Eq. (A1). Using the density of the Coulomb external potentials, we can constructing a sequence of Coulomb potentials, $v_{K}^{\text {Coul }}(\mathbf{r})$, that converges to $v(\mathbf{r})$, at least for the purposes of evaluating integrals like Eq. (A4). Based on this, what can we say about the sequence of Coulomb densities corresponding to $v_{K}^{\text {Coul }}(\mathbf{r}), \rho_{K}^{\text {Coul }}(\mathbf{r})$ ? Do they converge to the density in question?

In one sense, it seems likely that $\rho_{K}^{\text {Coul }}(\mathbf{r}) \rightarrow \rho(\mathbf{r})$. Specifically, if we regard the change in external potential, $v_{K}^{\text {Coul }}(\mathbf{r})-v(\mathbf{r})$, as a perturbation, we can express the change in density as

$$
\begin{aligned}
\rho_{K}^{\text {Coul }}(\mathbf{r})-\rho(\mathbf{r}) & =\int\left(\frac{\delta \rho(\mathbf{r})}{\delta v\left(\mathbf{r}^{\prime}\right)}\right)_{N}\left(v_{K}^{\text {Coul }}\left(\mathbf{r}^{\prime}\right)-v\left(\mathbf{r}^{\prime}\right)\right) d \mathbf{r}^{\prime} \\
& +\frac{1}{2} \iint\left(v_{K}^{\text {Coul }}\left(\mathbf{r}^{\prime \prime}\right)-v\left(\mathbf{r}^{\prime \prime}\right)\right)\left(\frac{\delta^{2} \rho(\mathbf{r})}{\left.\delta v\left(\mathbf{r}^{\prime \prime}\right) \delta v\left(\mathbf{r}^{\prime}\right)\right)_{N}\left(v_{K}^{\text {Coul }}\left(\mathbf{r}^{\prime}\right)-v\left(\mathbf{r}^{\prime}\right)\right) d \mathbf{r}^{\prime} d \mathbf{r}^{\prime \prime}+\ldots}\right.
\end{aligned}
$$

We expect the functional derivatives to be well-behaved functionals, so based on Eq. (A4), as $K$ becomes large, the corrections to the electron density should become increasing small (and the perturbation series should be rapidly convergent). In this sense, we expect that $\rho_{K}^{\text {Coul }}(\mathbf{r}) \rightarrow \rho(\mathbf{r})$.

There is also a sense in which the Coulomb densities may not converge to the target density: based on the discussion surrounding Eq. (A3), we expect that the derivatives of the 
Coulomb density may differ markedly from $\rho(\mathbf{r})$. Indeed this is the case: the Coulomb density, $\rho_{K}^{\text {Coul }}(\mathbf{r})$, has $K$ cusps, while the target density, $\rho(\mathbf{r})$, may lack cusps altogether. As $K$ becomes large, $\rho_{K}^{\text {Coul }}(\mathbf{r})$ becomes very jagged. Thus, while it may be true that

$$
\int\left|\rho_{K}^{\text {Coul }}(\mathbf{r})-\rho(\mathbf{r})\right| d \mathbf{r} \rightarrow 0
$$

it is probably not true that

$$
\int\left|\nabla \sqrt{\rho_{K}^{\text {Coul }}(\mathbf{r})-\rho(\mathbf{r})}\right|^{2} d \mathbf{r} \rightarrow 0
$$

This is the primary reason we preferred the Sobolev-type norm in Eq. (10). Exploring further, we expect that the Weisacker kinetic-energy bound,

$$
\int|\nabla \sqrt{\rho(\mathbf{r})}|^{2} d \mathbf{r}<T[\rho]<F^{\text {Coul }}[\rho]
$$

could be much larger for the jagged Coulomb density, $\rho_{K}^{\text {Coul }}(\mathbf{r})$, than it is for the target electron density, $\rho(\mathbf{r})$. Using the $\mathbb{L}^{1}$ distance (Eq. (A6)) to measure the distance between electron densities would be very problematic, because we would have two electron densities that were "close together" (in the sense of Eq. (A6)) that, owing to Eq. (A8), could have vastly different values for $F^{C o u l}[\rho]$. That is, if the $\mathbb{L}^{1}$ distance were used, then $F^{C o u l}[\rho]$ would be a discontinuous functional. Not only is it difficult to conceive of any practical way to construct approximate functional that are discontinuous, discontinuous functionals are necessarily nondifferentiable. Throughout DFT we assume that the functionals of interest are differentiable (in fact, we already implicitly assumed this in writing the variational identities in Eqs. (2) and (3)); practical considerations require, then, that our approximate functional at least be continuous.

The preceding analysis indicates that we must be very careful in how we choose to evaluate the "distance" between electron densities in defining these constrained search 
functionals. Unlike the simple $\mathbb{L}^{1}$ distance (Eq. (A6)), there seems to be no obvious reason why distance formula in Eq. (10) should be problematic. Even if this distance does prove problematic, however, there is no reason to suspect that another measure of the distance (perhaps one that depends more strongly on the similarity of the densities' cusps and/or asymptotic characteristics) would not suffice.

A necessary (and, for an appropriate choice of the distance, perhaps sufficient) condition for $F^{C o u l}[\rho]$ to be continuous is that $F^{C o u l}[\rho]$ be continuous on the subset of Coulomb densities. That is, for any $\varepsilon>0$, there exists a $\delta(\varepsilon)>0$ such that if two Coulomb densities, $\rho_{1}^{\text {Coul }}(\mathbf{r})$ and $\rho_{2}^{\text {Coul }}(\mathbf{r})$ satisfy

$$
\left\|\rho_{1}^{\text {Coul }}(\mathbf{r})-\rho_{2}^{\text {Coul }}(\mathbf{r})\right\|<\delta(\varepsilon)
$$

then

$$
\left|F^{\text {Coul }}\left[\rho_{1}^{\text {Coul }}\right]-F^{\text {Coul }}\left[\rho_{2}^{\text {Coul }}\right]\right|<\varepsilon
$$

Equation (A10) can be rewritten using Eq. (7),

$$
\left|E_{k_{1}}\left[v_{1}^{\text {Coul }}\right]-E_{k_{2}}\left[v_{2}^{\text {Coul }}\right]-\left(\int \rho_{1}^{\text {Coul }}(\mathbf{r}) v_{1}^{\text {Coul }}(\mathbf{r}) d \mathbf{r}-\int \rho_{2}^{\text {Coul }}(\mathbf{r}) v_{2}^{\text {Coul }}(\mathbf{r}) d \mathbf{r}\right)\right|<\varepsilon
$$

The biggest difference between this approach and the usual approaches to excited-state functionals is that the excitation levels of the two densities, $k_{1}$ and $k_{2}$, may be different. It is reasonable to assume that Coulomb densities that are close together will always have external potentials that are similar enough to ensure that the second term in (A11) is small. However, if Coulomb densities that are close together can have vastly different excitation levels, then we 
would still not expect the functional to be continuous. That is, our biggest concern regarding the continuity of $F^{C o u l}[\rho]$, is that there might exist two Coulomb densities, arbitrarily close together, that corresponded to very different levels of excitation; this may make it difficult to satisfy inequality (A11). Unfortunately, we do not know whether this occurs.

Possible problems associated with similar Coulomb densities corresponding to dissimilar levels of excitation are avoided when we use $F_{k}^{\text {Coul }}[\rho]$. In this case, continuity on the set of Coulomb densities dictates that for any $\varepsilon>0$, there exists a $\delta(\varepsilon)>0$ such that if the $k^{\text {th }}$ eigendensities of two distinct Coulomb systems, $\rho_{1}^{\text {Coul }}(\mathbf{r})$ and $\rho_{2}^{\text {Coul }}(\mathbf{r})$ satisfy inequality (A9), then

$$
\left|F_{k}^{\text {Coul }}\left[\rho_{1}^{\text {Coul }}\right]-F_{k}^{\text {Coul }}\left[\rho_{2}^{\text {Coul }}\right]\right|<\varepsilon
$$

This inequality can be replaced with

$$
\left|E_{k}\left[v_{1}^{\text {Coul }}\right]-E_{k}\left[v_{2}^{\text {Coul }}\right]-\left(\int \rho_{1}^{\text {Coul }}(\mathbf{r}) v_{1}^{\text {Coul }}(\mathbf{r}) d \mathbf{r}-\int \rho_{2}^{\text {Coul }}(\mathbf{r}) v_{2}^{\text {Coul }}(\mathbf{r}) d \mathbf{r}\right)\right|<\varepsilon
$$

Based on our discussion of the continuity of $F^{C o u l}[\rho]$, it seems likely that $F_{k}^{\text {Coul }}[\rho]$ is continuous, at least on the set of subset of densities associated with Coulomb systems. Since this is the case, the extension of $F_{k}^{\text {Coul }}[\rho]$ to non-Coulomb systems is expected to yield a wellbehaved functional.

The differentiability of density functionals is an issue that pervades density functional theory. ${ }^{10,63-70}$ Since our excited-state functionals include the ground-state functional as a special case, they exhibit the same derivative discontinuities as the conventional ground-state density functionals. It is often supposed, ${ }^{71-73}$ but it has not been rigorously proved, ${ }^{26,74}$ that the fundamental ground-state density functional is continuous. (The ensemble-constrained search 
functional ${ }^{75}$ is at least lower semicontinuous. ${ }^{74}$ ) All discussions of functional differentiability and continuity in DFT must be prefaced by the caveat that only "nice" densities and "nice" variations of the density are being; otherwise the functionals are manifestly discontinuous and nondifferentiable. Ideally the density will be strictly positive with compact (or essentially compact) support, and the variations will be local and "conventional."

Our functionals definitely have discontinuous derivatives at integer particle number, ${ }^{64,77}$ as can be inferred by extending ground-state arguments. ${ }^{64,66,78}$ For a given number of electrons, $N$, there are derivative discontinuities associated with degenerate states. ${ }^{66,79}$ The functionals might even be discontinuous here. Consider a fixed number of electrons $N$, and then adjust the positions of the point charges (alternatively, adjust the size of the point charges) so that one encounters a crossing between two potential energy surfaces, corresponding to the states labeled with $k$ and $k+1$. Very near the seam (or conical intersection) of the surfaces, the energy of the two states is almost the same but the components of the energy (the electron-external potential interaction energy of the two states and the values of $F_{k}^{\text {Coul }}[\rho]$ and $F_{k+1}^{\text {Coul }}[\rho]$ ) are usually different. On the other side of the seam, the roles of the $k$ and $k+1$ states are exchanged, therefore $F_{k}^{\text {Coul }}[\rho]$ changes discontinuously as a function of the position (and/or sizes) of the point charges. However, the electron density also changes discontinuously as a function of the position (and/or sizes) of the point charges at the surface crossing (because the response kernel diverges for degenerate states), so $F_{k}^{\text {Coul }}[\rho]$ might be continuous (though it generally will be nondifferentiable) along the seam of degenerate densities. Note that this issue is present already in the ground-state theory $(k=1){ }^{80}$ For the ground state theory, there are arguments that $F_{k=1}^{C o u l}[\rho]$ should be not only continuous, but differentiable in a certain generalized sense, with 
respect to constant- $N$ variations in the electron density. ${ }^{71-73}$ We suspect that similar arguments can be extended to excited states of the $F_{k}^{\text {Coul }}[\rho]$ functional, but probably not to $F^{C o u l}[\rho]$.

A few of the fundamental theorems presented here have already been introduced in the context of shape-functional theory, ${ }^{36,81,82}$ wherein the fundamental variable is not the electron density, but the density per particle, $\sigma(\mathbf{r})=\rho(\mathbf{r}) / N .^{83}$ (The shape function satisfies all of the preceding results because both the cusp conditions and the asymptotic condition, Eq. (6), depend only on the logarithmic derivative of the density, these properties are unaffected by the number of electrons.) However, the density-functional analogue of these results is to be preferred. If the Hohenberg-Kohn functional for the shape function, $F^{C o u l}[\sigma]$ is continuous, then the HohenbergKohn functionals for the electron density will also be continuous. However, the converse is not true. Insofar as practical application of these results will depend on the ability to construct good approximations to $F^{\text {Coul }}[\rho]$ or $F_{k}^{\text {Coul }}[\rho]$, it is advisable to focus efforts, at least initially, on the density functionals, as they are more likely to be continuous. 


\section{References:}

$1 \quad$ P. Hohenberg and W. Kohn, Phys.Rev. 136, B864 (1964).

$2 \quad$ W. Kohn and L. J. Sham, Phys.Rev. 140, A1133 (1965).

$3 \quad$ M. Levy, Int. J. Quantum Chem. 110, 3140 (2010).

4 H. Appel, E. K. U. Gross, and K. Burke, Phys. Rev. Lett. 90 (2003).

5 M. E. Casida, Journal of Molecular Structure-Theochem 914, 3 (2009).

6 M. E. Casida and D. P. Chong, in Recent Advances in Density Functional Methods. Part1. (World Scientific, Singapore, 1995), pp. 155.

7 M. Petersilka, U. J. Gossmann, and E. K. U. Gross, Phys. Rev. Lett. 76, 1212 (1996).

$8 \quad$ A. Nagy, Adv. Quantum Chem. 42, 363 (2003).

9 A. Nagy, M. Levy, and P. W. Ayers, in Chemical reactivity theory: A density functional view, edited by P. K. Chattaraj (Taylor and Francis, Boca Raton, 2009), pp. 121. J. P. Perdew and M. Levy, Phys. Rev. B 31, 6264 (1985).

A. K. Theophilou, Journal of Physics C 12, 5419 (1979).

A. K. Theophilou and N. I. Gidopoulos, Int. J. Quantum Chem. 56 (4), 333 (1995).

A. K. Theophilou, Int. J. Quantum Chem. 61, 333 (1997).

E. K. U. Gross, L. N. Oliveira, and W. Kohn, Phys. Rev. A 37, 2809 (1988).

L. N. Oliveira, E. K. U. Gross, and W. Kohn, Int. J. Quantum Chem. S24, 707 (1990).

W. Kohn, Phys. Rev. A 34 (2), 737 (1986).

M. Levy, Phys. Rev. A 52, R4313 (1995).

N. I. Gidopoulos, P. G. Papaconstantinou, and E. K. U. Gross, Phys. Rev. Lett. 88 (2002). M. Levy and A. Nagy, Phys. Rev. Lett. 83, 4361 (1999).

M. Levy and A. Nagy, Phys. Rev. A 59, 1687 (1999). 
A. Nagy and M. Levy, Phys. Rev. A 63, 052502 (2001).

P. W. Ayers, University of North Carolina at Chapel Hill, 2001.

V. Sahni and X. Y. Pan, Phys. Rev. Lett. 90, 123001 (2003).

P. W. Ayers and M. Levy, Phys. Rev. A 80, 012508 (2009).

T. Ziegler, M. Seth, M. Krykunov, J. Autschbach, and F. Wang, J. Chem. Phys. 130, $154102(2009)$.

E. H. Lieb, NATO ASI Series, Series B 123 (Density Functional Methods in Physics), 31 (1985).

A. Gorling, Phys. Rev. A 59, 3359 (1999).

R. Gaudoin and K. Burke, Phys. Rev. Lett. 93, 173001 (2004).

R. Gaudoin and K. Burke, Phys. Rev. Lett. 94, 029901 (2005).

P. Samal and M. K. Harbola, J. Phys. B 38, 3765 (2005).

P. Samal, M. K. Harbola, and A. Holas, Chem. Phys. Lett. 419, 217 (2006).

A. Nagy, Int. J. Quantum Chem. 70, 681 (1998).

T. Kato, Commun.Pure Appl.Math. 10, 151 (1957).

R. T. Pack and W. B. Brown, J. Chem. Phys. 45, 556 (1966).

E. Steiner, J. Chem. Phys. 39, 2365 (1963).

P. W. Ayers, Proc. Natl. Acad. Sci. 97, 1959 (2000).

A. Nagy and K. D. Sen, Chem. Phys. Lett. 332, 154 (2000).

A. Nagy and K. D. Sen, J. Phys. B 33, 1745 (2000).

A. Nagy and K. D. Sen, J. Chem. Phys. 115, 6300 (2001).

M. M. Morrell, R. G. Parr, and M. Levy, J. Chem. Phys. 62, 549 (1975).

M. Levy and R. G. Parr, J. Chem. Phys. 64, 2707 (1976). 
J. Katriel and E. R. Davidson, Proc. Natl. Acad. Sci. 77, 4403 (1980).

C. O. Almbladh and U. Von Barth, Phys. Rev. B 31, 3231 (1985).

S. Fournais, M. Hoffmann-Ostenhof, T. Hoffmann-Ostenhof, and T. O. Sorensen, in Quantum Few-Body Systems, edited by J. S. Moller and D. Fedorov (2008), Vol. 998, pp. 70.

M. Levy, J. P. Perdew, and V. Sahni, Phys. Rev. A 30, 2745 (1984).

D. Vanfleteren, D. Van Neck, P. W. Ayers, R. C. Morrison, and P. Bultinck, J. Chem. Phys. 130, 194104 (2009).

B. G. Giraud, K. Kato, A. Ohnishi, and S. M. A. Rombouts, Phys. Lett. B 652, 69 (2007).

D. L. Whitenack and A. Wasserman, Phys. Rev. Lett. 107, 163002 (2011).

P. Samal and M. K. Harbola, J. Phys. B 39, 4065 (2006).

M. K. Harbola and P. Samal, Journal of Physics B-Atomic Molecular and Optical Physics 42, 015003 (2009).

M. K. Harbola, M. Shamim, P. Samal, M. Rahaman, S. Ganguly, and A. Mookerjee, in Computational Methods in Science and Engineering, Vol 1 - Advances in Computational Science, edited by G. Maroulis and T. E. Simos (2009), Vol. 1108, pp. 54.

M. Hemanadhan and M. K. Harbola, Journal of Molecular Structure-Theochem 943, 152 (2010).

V. N. Glushkov and X. Assfeld, J. Chem. Phys. 132, 204106 (2010).

M. Shamim and M. K. Harbola, Journal of Physics B-Atomic Molecular and Optical Physics 43, 215002 (2010).

V. N. Staroverov and V. N. Glushkov, J. Chem. Phys. 133, 244104 (2010).

F. Tasnadi and A. Nagy, Chem. Phys. Lett. 366, 496 (2002). 
F. Tasnadi and A. Nagy, J. Chem. Phys. 119, 4141 (2003).

There is also a constructive proof. Select $\mathrm{K}$ spheres in the space of interest. By choosing appropriate point charges in the spheres, we can approximate the external potential outside the spheres to any desired accuracy, which is just the idea behind multipole expansions. Next, increase the number of spheres but decrease the size of the spheres, and repeat the approximation process. Continuing in this fashion, the spheres shrink to zero size and one eventually finds that the volume of space in which the external potential is inaccurately rendered (due to the presence of the approximating point charges) is a set of measure zero. This method of proof relies on the fact that any countable subset of the real numbers has measure zero.

A. J. Cohen, P. Mori-Sanchez, and W. T. Yang, Science 321, 792 (2008).

P. W. Ayers and M. Levy, J. Chem. Sci. 117, 507 (2005).

P. W. Ayers, S. Golden, and M. Levy, J. Chem. Phys. 124, 054101 (2006).

P. W. Ayers and P. Fuentealba, Phys. Rev. A 80, 032510 (2009).

Many external potentials that cannot be expressed in this form can still be approximated, to arbitrary precision, by an external potential with this form. and repeat the approxination process. Continuing in this fashion, the spheres shink to

A. J. Cohen, P. Mori-Sanchez, and W. T. Yang, Science 321, 792 (2008).

J. P. Perdew, R. G. Parr, M. Levy, and J. L. Balduz, Jr., Phys. Rev. Lett. 49, 1691 (1982).

J. P. Perdew and M. Levy, Phys. Rev. Lett. 51, 1884 (1983).

W. T. Yang, Y. K. Zhang, and P. W. Ayers, Phys. Rev. Lett. 84, 5172 (2000).

J. P. Perdew and M. Levy, Phys. Rev. B 56, 16021 (1997).

E. Sagvolden, J. P. Perdew, and M. Levy, Phys. Rev. A 79, 026501 (2009).

P. Mori-Sanchez, A. J. Cohen, and W. T. Yang, Phys. Rev. Lett. 102, 066403 (2009).

P. E. Lammert, Int. J. Quantum Chem. 107, 1943 (2007). 
H. Englisch and R. Englisch, Phys.Status Solidi B 123, 711 (1984).

H. Englisch and R. Englisch, Physica Status Solidi B 124, 373 (1984).

P. W. Ayers, Phys. Rev. A 73, 012513 (2006).

E. H. Lieb, Int. J. Quantum Chem. 24, 243 (1983).

S. M. Valone, J. Chem. Phys. 73, 4653 (1980).

H. Englisch, H. Fieseler, and A. Haufe, Phys. Rev. A 37, 4570 (1988).

Y. K. Zhang and W. T. Yang, Theor. Chem. Acc. 103, 346 (2000).

P. W. Ayers, J. Math. Chem. 43, 285 (2008).

M. Levy, Phys. Rev. A 26, 1200 (1982).

R. Baer, Phys. Rev. Lett. 104 (7) (2010).

F. De Proft, P. W. Ayers, K. D. Sen, and P. Geerlings, J. Chem. Phys. 120, 9969 (2004).

P. W. Ayers, Phys. Rev. A 71, 062506 (2005).

R. G. Parr and L. J. Bartolotti, J.Phys.Chem. 87, 2810 (1983). 